Elizabeth Loehrer ${ }^{1}$ Meike W. Vernooij, ${ }^{1,2}$ M. Arfan $1 \mathrm{kram}^{1,2,3,3}$

'Department of Epidemiology, Erasmus MC University Medical Center, 3000 CA, Rotterdam, ZH, Netherlands 2Department of Radiology, Erasmus MC University Medical Center, 3000 CA, Rotterdam, ZH, Netherlands ${ }^{3}$ Department of Neurology, Erasmus MC University Medical Center, 3000 CA, Rotterdam, ZH, Netherlands

Received 09 May 2014 accepted 29 May 2014

\section{CEREBRAL MICROBLEEDS: SPATIAL DISTRIBUTION IMPLICATIONS}

\section{Abstract}

Cerebral microbleeds are considered an imaging marker of cerebral small vessel disease. The location of microbleeds is thought to reflect the underlying pathology. Microbleeds in the deep and infratentorial region are thought to reflect hypertensive arteriopathy whereas lobar microbleeds are associated clinically with cerebral amyloid angiopathy (CAA). Aside from patient populations, microbleeds are frequently observed in seemingly asymptomatic populations. Moreover, many elderly, both in clinical and preclinical populations, have multiple coexisting pathologies in their brains, which complicates the interpretation of cerebral microbleeds, especially early in the clinical course. In this commentary, we discuss the influence of the strongest genetic risk factor for CAA, Apolipoprotein E (APOE), in the spatial distribution of microbleeds, and we additionally address issues in interpretation and implication of the location of microbleeds in clinical and asymptomatic populations.

\section{Keywords}

- Cerebral microbleeds $\cdot$ Cerebral small vessel disease $\cdot$ Cerebrovascular disease $\cdot$ Cerebral amyloid angiopathy

- Neuroradiology $\cdot \mathrm{MRI} \cdot$ Imaging markers

(c) Versita Sp. z o.o.
Cerebral microbleeds, detected using magnetic resonance imaging (MRI), are considered an imaging marker of cerebral small vessel disease [1]. Microbleeds can occur throughout the brain, and their location is thought to reflect the underlying pathology [1]. Typically, microbleeds in the deep and infratentorial regions are thought to reflect hypertensive arteriopathy $[1,2]$. In contrast, lobar cerebral microbleeds are associated with cerebral amyloid angiopathy (CAA), which is a build-up of beta-amyloid in the cortical and subcortical vessels of the brain that can lead to either small infarctions or vessel rupture [3-6]. When vessels rupture, the results are small cerebral microbleeds or - clinically more devastating large, symptomatic intracerebral hemorrhage (ICH). In addition to patients with lobar ICH, CAA frequently coexists with Alzheimer's disease (AD) pathology [7]. Like in $A D$, the strongest known genetic risk factor for $C A A$ and CAA-related hemorrhages is Apolipoprotein $E$ (APOE), but, in contrast to $A D$, both the APOE e4 and the APOE e2 variant alleles increase risk compared to the wildtype e3e3.

Within individual patients with CAA, the distribution of vascular beta-amyloid appears patchy, and the resulting hemorrhagic outcomes tend to cluster [8]. Though CAA can occur in any lobar region of the brain [6], the posterior lobar regions seem more prone to severe vascular beta-amyloid depositions $[6,9]$. Likewise, the resulting CAA-related $\mathrm{ICH}$ and cerebral microbleeds have primarily posterior lobar distributions, with the occipital lobe, in particular, exceptionally vulnerable to hemorrhagic outcomes $[8,10,11]$. Several elegant studies in CAA patients have provided strong evidence that microbleeds occur in areas of high loads of vascular amyloid [2,4,12].

Aside from patient populations, microbleeds are frequently observed in seemingly asymptomatic, general population [13-16]. The predominantly posterior distribution of lobar microbleeds has also been reported in these populations $[13,17]$. However, the underlying pathologies of microbleeds in this group is still largely unconfirmed via amyloid imaging or autopsy, because these techniques are not widely available in the general population. One case-control study of $A D$ and preclinical$A D$ patients demonstrated that healthy controls with lobar cerebral microbleeds had significantly higher loads of beta-amyloid than those without lobar microbleeds [11], and that incident lobar microbleeds in healthy controls are associated with both carriership of APOE e4 allele and higher load of beta-amyloid [18]. Moreover, the healthy controls who were e4 carriers had higher amyloid retention compared to those who were non-carriers [19]. In a recent paper, posterior distribution of lobar microbleeds within the entire communitydwelling Rotterdam Study population was found, but those among this population, who were carriers of the APOE e2 or e4 alleles, had microbleeds that occurred closer together and had an ever higher proportion of microbleeds in the occipital lobe compared to persons with APOE e3e3 genotype [20].

Though APOE e2 and e4 alleles are both associated with presence of CAA and risk of adverse outcomes due to CAA [21,22], each allele is associated with the pathology differently. Specifically, e4 allele is suggested to relate to CAA type 1, which includes amyloid depositions in the capillaries, whereas e2 allele is overrepresented among CAA type 2 , which is devoid of amyloid depositions in the capillaries [23]. Moreover, the APOE e2 and e4 variants are thought to lead to hemorrhagic outcomes

*E-mail:m.a.ikram@erasmusmc.nl 
through different mechanisms. Studies in CAA and $A D$ patients have shown that while the e4 allele carriers are shown to accumulate more beta-amyloid and faster, leading to a thickening and eventual rupture of vessel walls, e2 carriers are thought to have increased vessel fragility, leading to easier rupture of vessels without an increased amyloid burden compared to normal genotype $[21,22,24-26]$. Thus, these two alleles seem to result in distinct pathways, both of which are more susceptible to hemorrhagic outcomes compared to the wild-type genotype. The described posterior distribution of beta-amyloid deposition [6,9], lobar ICH [8], and microbleeds [8] in CAA seems to suggest that the posterior circulating territory is disproportionately affected compared to other vascular territories, though the specific reason for this remains unclear. Taken together, our recent findings are likely representing a particularly vulnerable posterior circulating territory among all persons coupled with increased susceptibility to adverse outcomes among APOE risk allele carriers, leading to even more events in that region than we observe in the wild-type APOE. When combined with findings from other studies, the available evidence supports the notion that APOE has a subtle effect on the pathology underlying lobar microbleeds in both patient and asymptomatic general populations.

Clinically, only persons with multiple strictly lobar bleeds, that is, microbleeds or $\mathrm{ICH}$ only in the lobar location, are included in the diagnostic criteria of (probable) CAA [27]. The reality is that many elderly, both in clinical and preclinical populations, have multiple coexisting pathologies in the brain [28-31]. When microbleeds occur in both the lobar and deep and/or infratentorial regions in the same person, the underlying pathology responsible for the bleeds is less clear. Mixed lobar/deep/infratentorial microbleeds have been reported in patient and population-based settings, and they are typically attributed to hypertensive arteriopathy in situations when the driving pathology is not known $[1,13,15,30,32,33]$. In the population-based Rotterdam Study, we have shown that even persons with any lobar microbleeds (with or without microbleeds in other locations) show a posterior predominant distribution similar to CAA population distributions and are likely the result of underlying CAA $[17,20]$. Our most recent work also showed that among all microbleeds, incident microbleeds occurred in close proximity to prevalent microbleeds within participants, particularly among APOE risk allele carriers [20]. Though the microbleeds in this analysis were primarily lobar, we did observe clustering between microbleeds that technically fell on opposite sides of regional boundaries, i.e., deep and lobar (Figure 1) [20]. We can imagine that microbleeds that occur very close together share the same pathology (or pathologies), and reflect vulnerability in a specific location, even if they are technically in different brain regions. Though this specific scenario has not been described extensively in literature, there is some evidence that the typical strict division between lobar and deep microbleeds might need some rethinking, or at the very least, further investigation. For example, though APOE variant alleles have typically been studied in regard to lobar
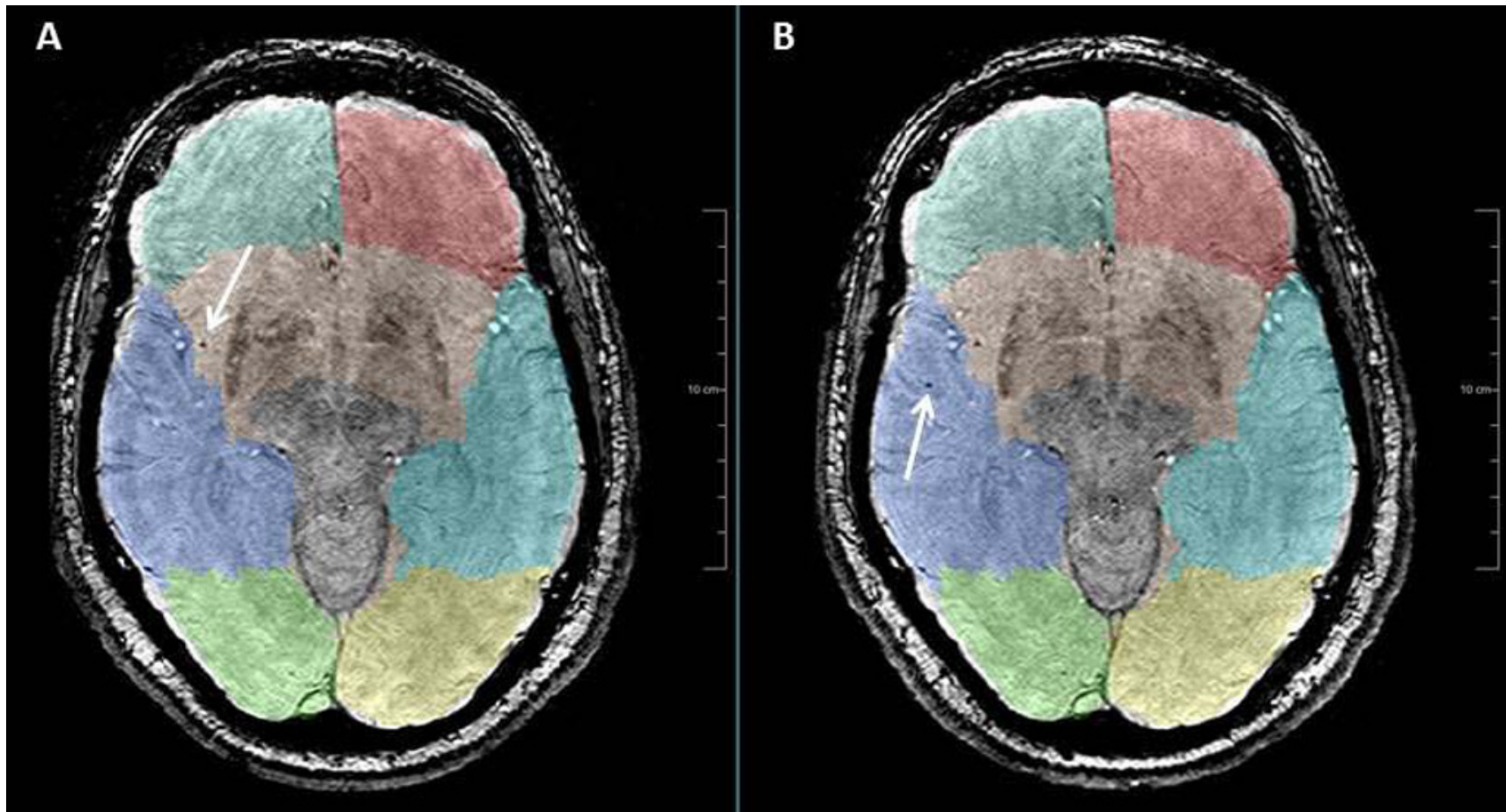

Figure 1. Magnetic resonance images of baseline (A) and follow-up (B) scans of one participant, which demonstrates that microbleeds can occur close together but on opposite sides of the lobar/deep-border. In this instance, the prevalent microbleed (arrow in A) would be rated as "deep", whereas the incident microbleed (arrow in B) would be rated as "lobar". The question is whether these microbleeds occurred due to different pathologies, or due to one causal factor. 
hemorrhages and amyloid related pathologies (i.e. CAA and $A D$ ), two recent meta-anlaysis have shown that carriership of the APOE e4 allele increases risk of both lobar and deep microbleeds [34,35].

In the same light, cerebellar microbleeds also remain difficult to classify. Infratentorial microbleeds, including cerebellar microbleeds, are usually attributed to hypertensive arteriopathy [1,2,36]. However, cerebellar microbleeds are also allowed to be present for diagnosis of probable CAA, though betaamyloid deposition is thought to be present in the infratentorial regions only in the severe cases of CAA [36,37]. At least one study of ICH patients observed cerebellar microbleeds in persons with probable CAA, persons with strictly deep $\mathrm{ICH}$, and persons with mixed deep and lobar bleeds, highlighting the fact that these microbleeds do not seem to be specific to a particular pathology [30]. When cerebellar microbleeds are observed in the general population, they are usually attributed to hypertensive risk factors [15], but the cause of these bleeds may be misattributed, particularly when they are in brains with many microbleeds (both lobar and deep). In clinical settings, the difficulty in interpreting mixed microbleeds is transposed to the interpretation of intracerebral hemorrhages that occur at the border of deep and lobar regions, or transgress this border. Therefore, the clinical management of these hemorrhages is not straightforward. Ideally, microbleeds could be used as a marker of underlying pathology and lead to intervention prior to a major clinical event. At the moment, the main challenge with interpreting microbleeds is that it is unclear if a singular, advanced pathology is responsible for bleeds in multiple regions, whether mixed pathologies are present and operating concurrently within the same brain, or whether mixed pathologies interact with each other, resulting in a distinct causal mechanism for hemorrhagic outcomes.
Understanding of the causes and relevance of the spatial distribution of cerebral microbleeds is still lacking in both patient and asymptomatic populations. Though clinical studies have provided ample knowledge on microbleeds to date, they tend to focus on severe and homogenous patients. More typical, elderly with mixed pathologies, are an important group to study as they could lend crucial insight into our understanding of the progression of cerebral small vessel disease to symptomatic outcomes. As new minimally-invasive amyloid imaging techniques start to become more accessible, researchers can address many remaining questions regarding the involvement of betaamyloid in lobar and non-lobar microbleeds in asymptomatic persons. Population-based studies are optimal to study asymptomatic persons with mixed microbleeds to untangle the causal mechanism(s) of these bleeds, and to identify the predominant implications of microbleeds that appear early in the clinical course.

\section{References}

[1] Greenberg S.M., Vernooij M.W., Cordonnier C., Viswanathan A., AlShahi Salman R., Warach S., et al., Cerebral microbleeds: a guide to detection and interpretation, Lancet Neurol., 2009, 8, 165-174

[2] Fazekas F., Kleinert R., Roob G., Kleinert G., Kapeller P., Schmidt R., et al., Histopathologic analysis of foci of signal loss on gradient-echo T2*-weighted MR images in patients with spontaneous intracerebral hemorrhage: evidence of microangiopathy-related microbleeds, AJNR Am. J. Neuroradiol., 1999, 20, 637-642

[3] Schrag M., McAuley G., Pomakian J., Jiffry A., Tung S., Mueller C., et al., Correlation of hypointensities in susceptibility-weighted images to tissue histology in dementia patients with cerebral amyloid angiopathy: a postmortem MRI study, Acta Neuropathol., 2010, 119, 291-302

[4] Dierksen G.A., Skehan M.E., Khan M.A., Jeng J., Nandigam R.N., Becker J.A., et al., Spatial relation between microbleeds and amyloid deposits in amyloid angiopathy, Ann. Neurol., 2010, 68, 545-548

[5] Viswanathan A., Greenberg S.M., Cerebral amyloid angiopathy in the elderly, Ann. Neurol., 2011, 70, 871-880

[6] Vinters H.V., Cerebral amyloid angiopathy. A critical review, Stroke, $1987,18,311-324$

[7] Thal D.R., Griffin W.S., de Vos R.A., Ghebremedhin E., Cerebral amyloid angiopathy and its relationship to Alzheimer's disease, Acta Neuropathol., 2008, 115, 599-609

[8] Rosand J., Muzikansky A., Kumar A., Wisco J.J., Smith E.E., Betensky R.A., et al., Spatial clustering of hemorrhages in probable cerebral amyloid angiopathy, Ann. Neurol., 2005, 58, 459-462

[9] Johnson K.A., Gregas M., Becker J.A., Kinnecom C., Salat D.H., Moran E.K., et al., Imaging of amyloid burden and distribution in cerebral amyloid angiopathy, Ann. Neurol., 2007, 62, 229-234

[10] Pettersen J.A., Sathiyamoorthy G., Gao F.Q., Szilagyi G., Nadkarni N.K., St George-Hyslop P., et al., Microbleed topography, leukoaraiosis, and cognition in probable Alzheimer disease from the Sunnybrook dementia study, Arch. Neurol., 2008, 65, 790-795

[11] Yates P.A., Sirisriro R., Villemagne V.L., Farquharson S., Masters C.L., Rowe C.C., et al., Cerebral microhemorrhage and brain beta-amyloid in aging and Alzheimer disease, Neurology, 2011, 77, 48-54

[12] Gurol M.E., Dierksen G., Betensky R., Gidicsin C., Halpin A., Becker A., et al., Predicting sites of new hemorrhage with amyloid imaging in cerebral amyloid angiopathy, Neurology, 2012, 79, 320-326

[13] Sveinbjornsdottir S., Sigurdsson S., Aspelund T., Kjartansson O., Eiriksdottir G., Valtysdottir B., et al., Cerebral microbleeds in the population based AGES-Reykjavik study: prevalence and location, J. Neurol. Neurosurg. Psychiatry, 2008, 79, 1002-1006

[14] Jeerakathil T., Wolf P.A., Beiser A., Hald J.K., Au R., Kase C.S., et al., Cerebral microbleeds: prevalence and associations with cardiovascular risk factors in the Framingham Study, Stroke, 2004, 35, 1831-1835

[15] Poels M.M., Vernooij M.W., Ikram M.A., Hofman A., Krestin G.P., van der Lugt A., et al., Prevalence and risk factors of cerebral microbleeds: an update of the Rotterdam scan study, Stroke, 2010, 41, S103-106 
[16] Vernooij M.W., van der Lugt A., Ikram M.A., Wielopolski P.A., Niessen W.J., Hofman A., et al., Prevalence and risk factors of cerebral microbleeds: the Rotterdam Scan Study, Neurology, 2008, 70, 12081214

[17] Mesker D.J., Poels M.M., Ikram M.A., Vernooij M.W., Hofman A., Vrooman H.A., et al., Lobar distribution of cerebral microbleeds: the Rotterdam Scan Study, Arch. Neurol., 2011, 68, 656-659

[18] Yates P.A., Desmond P.M., Phal P.M., Steward C., Szoeke C., Salvado O., et al., Incidence of cerebral microbleeds in preclinical Alzheimer disease, Neurology, 2014, 82, 1266-1273

[19] Rowe C.C., Ellis K.A., Rimajova M., Bourgeat P., Pike K.E., Jones G., et al., Amyloid imaging results from the Australian Imaging, Biomarkers and Lifestyle (AIBL) study of aging, Neurobiol. Aging, 2010, 31, 12751283

[20] Loehrer E., Ikram M.A., Akoudad S., Vrooman H.A., van der Lugt A., Niessen W.J., et al., Apolipoprotein $E$ genotype influences spatial distribution of cerebral microbleeds, Neurobiol. Aging, 2014, 35, 899905

[21] Greenberg S.M., Rebeck G.W., Vonsattel J.P., Gomez-Isla T.,Hyman B.T., Apolipoprotein E epsilon 4 and cerebral hemorrhage associated with amyloid angiopathy, Ann. Neurol., 1995, 38, 254-259

[22] Greenberg S.M., Vonsattel J.P., Segal A.Z., Chiu R.I., Clatworthy A.E., Liao A., et al., Association of apolipoprotein $E$ epsilon2 and vasculopathy in cerebral amyloid angiopathy, Neurology, 1998, 50, 961-965

[23] Thal D.R., Ghebremedhin E., Rüb U., Yamaguchi H., Del Tredici K., Braak H., Two types of sporadic cerebral amyloid angiopathy, J. Neuropathol. Exp. Neurol., 2002, 61, 282-293

[24] Schmechel D.E., Saunders A.M., Strittmatter W.J., Crain B.J., Hulette C.M., Joo S.H., et al., Increased amyloid beta-peptide deposition in cerebral cortex as a consequence of apolipoprotein $\mathrm{E}$ genotype in late-onset Alzheimer disease, Proc. Natl. Acad. Sci. USA, 1993, 90, 9649-9653

[25] McCarron M.O., Nicoll J.A., Stewart J., Ironside J.W., Mann D.M., Love S., et al., The apolipoprotein E epsilon2 allele and the pathological features in cerebral amyloid angiopathy-related hemorrhage, J. Neuropathol. Exp. Neurol., 1999, 58, 711-718
[26] Grimmer T., Tholen S., Yousefi B.H., Alexopoulos P., Forschler A., Forstl $\mathrm{H}$. , et al., Progression of cerebral amyloid load is associated with the apolipoprotein E e4 genotype in Alzheimer's disease, Biol. Psychiatry, 2010, 68, 879-884

[27] Knudsen K.A., Rosand J., Karluk D.,Greenberg S.M., Clinical diagnosis of cerebral amyloid angiopathy: validation of the Boston criteria, Neurology, 2001, 56, 537-539

[28] Jellinger K.A., Pathology and pathogenesis of vascular cognitive impairment-a critical update, Front. Aging Neurosci., 2013, 5, 17

[29] Schneider J.A., Bennett D.A., Where vascular meets neurodegenerative disease, Stroke, 2010, 41 (Suppl. 10), S144-146

[30] Smith E.E., Nandigam K.R., Chen Y.W., Jeng J., Salat D., Halpin A., et al., MRI markers of small vessel disease in lobar and deep hemispheric intracerebral hemorrhage, Stroke, 2010, 41, 1933-1938

[31] Thal D.R., Grinberg L.T., Attems J., Vascular dementia: different forms of vessel disorders contribute to the development of dementia in the elderly brain, Exp. Gerontol., 2012, 47, 816-824

[32] Romero J.R., Preis S.R., Beiser A., Decarli C., Viswanathan A., MartinezRamirez S., et al., Risk factors, stroke prevention treatments, and prevalence of cerebral microbleeds in the framingham heart study, Stroke, 2014, 45, 1492-1494

[33] Olazarán J., Ramos A., Boyano I., Alfayate E., Valentí M., Rábano A., et al., Pattern of and risk factors for brain microbleeds in neurodegenerative dementia, Am. J. Alzheimers Dis. Other Demen., $2014,29,263-269$

[34] Schilling S., DeStefano A.L., Sachdev P.S., Choi S.H., Mather K.A., DeCarli C.D., et al., APOE genotype and MRI markers of cerebrovascular disease: systematic review and meta-analysis, Neurology, 2013, 81, 292-300

[35] Maxwell S.S., Jackson C.A., Paternoster L., Cordonnier C., Thijs V., AlShahi Salman R., et al., Genetic associations with brain microbleeds: Systematic review and meta-analyses, Neurology, 2011, 77, 158-167

[36] Werring D.J., Cerebral microbleeds: pathophysiology to clinical practice, Cambridge, Cambridge University Press, 2011

[37] Thal D.R., Rüb U., Orantes M., Braak H., Phases of amyloid betadeposition in the human brain and its relevance for the development of Alzheimer's disease, Neurology, 2002, 58, 1791-1800 